\title{
Entre el activismo y la mediatización: encuadres de partidos y prensa en la campaña catalana de 2017
}

\section{Between activism and mediatization: parties and media's frames in the Catalan campaign of 2017}

\author{
Carratalá, A. y Palau-Sampio, D. ${ }^{1}$ \\ Recibido: 05-04-2019 - Aceptado: 9-08-2019 \\ DOI: https://doi.org/10.26441/RC18.2-2019-A4
}

\begin{abstract}
RESUMEN: Los estudios sobre la cobertura periodística de las campañas electorales apuntan a una creciente mediatización. Este trabajo busca corroborar si la tendencia también se da en circunstancias excepcionales, como las de las elecciones autonómicas de Cataluña en 2017. La investigación compara los encuadres presentados por seis periódicos (El País, El Mundo, La Vanguardia, El Periódico de Catalunya, Punt Avui y Ara) con los adoptados por los siete partidos políticos representados en el parlamento autonómico (JuntsXCat, ERC, PSC, PP, Ciudadanos, En Comú-Podem y CUP). Los resultados indican que los encuadres simbólicos propios de la acción colectiva dominaron en el mensaje de los partidos, aunque la lógica mediática volvió a ser hegemónica en términos globales.
\end{abstract}

Palabras clave: comunicación política; información política; framing; mediatización; campaña electoral; movilización.

ABSTRACT: Research on media coverage of electoral campaigns point to an increasing mediatization. This paper seeks to corroborate whether the trend also takes place in exceptional circumstances, such as those surrounding the regional elections in Catalonia in 2017. The analysis compares the frames observed in six newspapers (El País, El Mundo, La Vanguardia, El Periódico de Catalunya, Punt Avui and Ara) with those adopted by the seven political parties represented in the autonomous parliament (JuntsXCat, ERC, PSC, PP, Ciudadanos, En ComúPodem and CUP). The results indicate that the symbolic frames characteristic of collective action were dominant in the political discourses, although the media logic once again became hegemonic in global terms.

Keywords: political communication, political information, framing, mediatization, electoral campaign, mobilization.

\footnotetext{
${ }^{1}$ Adolfo Carratalá es Doctor en Periodismo por la Universitat de València y Profesor de Teoría e Historia del Periodismo y Periodismo de Sociedad y Cultura en la Facultad de Filología, Traducción y Comunicación de la misma universidad. adolfo.carratala@uv.es, http://orcid.org/0000-0002-9865-9246
}

Dolors Palau-Sampio es Doctora en Periodismo por la Universitat Autònoma de Barcelona y Profesora Titular de Periodismo en la Facultad de Filología, Traducción y Comunicación de la Universitat de València. dolors.palau@uv.es, http://orcid.org/0000-0001-9051-0239 


\section{Introducción ${ }^{2}$}

Las campañas electorales constituyen un fenómeno de máxima relevancia en el estudio de las relaciones entre la esfera política y la esfera mediática. Las investigaciones realizadas en esta área centran su interés en esclarecer el grado de influencia que un campo ejerce sobre el otro, para determinar si son los medios de comunicación o los actores políticos quienes lideran el establecimiento de la agenda y las estrategias discursivas durante los decisivos días previos a las elecciones. En los últimos años, el análisis de la comunicación electoral ha evidenciado una clara hegemonía de la lógica mediática sobre la política, como una expresión más del proceso de mediatización que afecta a la actividad de partidos e instituciones. Este liderazgo de las estrategias propias de los medios en el diseño de los discursos de los partidos implica una significativa apuesta por abrirse paso con facilidad en la arena mediática, aunque la creciente desafección y desconfianza por la política tradicional no asegure que ese estilo comunicativo contribuya, finalmente, a la movilización ciudadana.

La campaña que precedió a las elecciones catalanas de diciembre de 2017 reunió una serie de circunstancias que la convirtieron en un proceso excepcional: la votación fue convocada por el gobierno central una vez suspendida la autonomía del territorio tras la declaración de independencia realizada por el parlamento regional; la campaña tuvo lugar en fechas inusuales, anteriores a las fiestas de Navidad; el escenario se encontraba altamente polarizado, tras el resultado de las elecciones celebradas un par de años antes; dos de los cabezas de lista independentistas, Carles Puigdemont y Oriol Junqueras, no pudieron participar físicamente en la campaña por encontrarse huido en Bruselas y encarcelado, respectivamente; y, por primera vez, las encuestas apuntaban a que un partido no independentista y liderado por una mujer, Ciudadanos, podría resultar ganador de los comicios. Esta investigación indaga en si, en una campaña electoral de características tan singulares y carácter tan decisivo, partidos políticos y medios mantuvieron la lógica mediática en sus encuadres de comunicación electoral o, en cambio, favorecieron enfoques programáticos o de carácter simbólico con voluntad movilizadora.

\section{Marco teórico}

\subsection{Discurso electoral y mediatización política}

A pesar de que el fenómeno de la llamada campaña permanente (Blumenthal, 1980) implica una cada vez más difícil distinción de la etapa en que la comunicación política se estructura en torno a objetivos electorales, el periodo oficial de campaña que precede a una votación continúa revelándose como un momento decisivo para el estudio de las estrategias y discursos de partidos políticos y medios de comunicación. Aunque aproximadamente solo un tercio del electorado afirma aprovechar este tiempo para informarse de los programas de los partidos o coaliciones, conocer mejor a los candidatos u observar las diferencias entre unos partidos y otros (Berrocal \& Fernández, 2006), los estudios demuestran que la actividad desarrollada en campaña puede resultar decisiva para persuadir, sobre todo, a votantes inseguros o volátiles, y especialmente por parte de nuevos partidos (Barreiro Rivas, Pereira López \& García Hípola, 2015). Conscientes de esa oportunidad, las formaciones políticas destinan numerosos recursos a la gestión de la

\footnotetext{
${ }^{2}$ Este trabajo se enmarca en el Proyecto de I+D+i «Estrategias, agendas y discursos en las cibercampañas electorales: medios de comunicación y ciudadanos» (referencia CSO2016-77331-C2-1-R), concedido por el Ministerio de Economía y Competitividad para el período 2017-2020.
} 
comunicación en campaña (Farrell \& Schmitt-Beck, 2002), cada vez más profesionalizada de la mano del marketing comercial (Paniagua Rojano, 2004). Esta voluntad por hacer de la comunicación electoral una herramienta lo más eficaz posible debe interpretarse a partir de la lectura de la campaña como una batalla simbólica en la que los diferentes partidos concurrentes tratan de hacer que sus mensajes y discursos logren acceder con éxito a la arena pública (Valera-Ordaz, Carratalá \& Palau-Sampio, 2017), cuya gestión todavía se encuentra en buena medida bajo el control de los medios de comunicación tradicionales pese al creciente uso de las redes sociales.

La ventaja electoral deseada por las formaciones políticas ya no es buscada a través de la mera oferta de propuestas o medidas sobre los asuntos que cada partido entiende cruciales en el marco político y social en el que actúa, sino que las organizaciones tratan de alcanzarla por medio de conseguir un espacio preferente y favorable a su imagen en los medios de comunicación, pues estos suponen un canal esencial por el que la ciudadanía puede acceder al conocimiento y evaluación de los representantes públicos (RodríguezVirgili, López-Escobar \& Tolsá, 2012). Con el objetivo de asegurarse un espacio en el foro mediático, durante los últimos años los actores políticos han ido adaptando sus expresiones y actuaciones a las reglas y códigos propios de la actividad de los medios de comunicación, dando lugar a una de las más evidentes manifestaciones del fenómeno conocido como mediatización política (Davis, 2007; Mazzoleni \& Schulz, 2009; Strömbäck, 2008, 2011).

Esta progresiva adopción de los principios y códigos preferidos por los medios de comunicación ha supuesto que muchas de las actuaciones públicas de los actores políticos no persigan otra finalidad que ser foco de atención por parte de los periodistas. Desde los actos de campaña, que deben ser contemplados como media events (Dayan \& Katz, 1992) pues no tendrían sentido si no son objeto de cobertura mediática, hasta el propio discurso político, cuyos mensajes quedan enmarcados de acuerdo a los encuadres que los medios privilegian en el seguimiento de la actualidad política. Diferentes investigaciones (Berganza, 2008; De Vreese \& Semetko, 2002; Patterson, 1993) han puesto de manifiesto cómo la cobertura de la información política, sobre todo en periodo electoral, ha favorecido encuadres como el estratégico, centrado en las tácticas y planes de campaña, así como en las motivaciones y personalidades de los líderes, o el de juego, que representa la dinámica política como competición mediante el lenguaje de ganadores y perdedores y con metáforas propias del deporte y la guerra (Aalberg, Strömback \& De Vreese, 2011). Junto con ellos, otros encuadres como el de conflicto o el de interés humano también han ido ganando peso, en una operación dirigida a la promoción del infoentretenimiento en la cobertura política (Echevarría, 2017), dominante en la esfera televisiva (Peris Blanes, Llorca-Abad, Sánchez Castillo \& López-Rico, 2017).

Estas fórmulas de cobertura mediática no solo favorecen el cinismo de la ciudadanía hacia la política, especialmente a partir del encuadre estratégico (Cappella \& Jamieson, 1996; Valentino, Beckmann \& Buhr, 2001; De Vreese, 2005), sino que también implican desplazar del discurso informativo el contenido político de fondo, omitiendo propuestas sobre problemas públicos y medidas concretas ofrecidas por los partidos, es decir, los temas programáticos que debieran ocupar el mensaje de las formaciones en contienda electoral y ser también los hegemónicos en la información política (Iyengar, 1991). Sin embargo, el fenómeno de mediatización apunta a que la responsabilidad de este proceso que privilegia la lógica de los medios sobre la política no debe caer en exclusiva en los primeros. Como indican investigaciones recientes (Blumler \& Esser, 2018), los actores políticos también serían impulsores del proceso de mediatización. Esta perspectiva centrada en el actor político como motor de estas estrategias se basaría en la introducción 
del profesionalismo en las campañas electorales por parte de los partidos políticos, sobre todo con el objetivo de mejorar la gestión de las informaciones, conscientes de su dependencia respecto al gatekeeping de los medios (Meyer, Haselmayer \& Wagner, 2017). En este sentido, el estudio de los encuadres de campaña durante las elecciones generales de 2015 en España (Palau-Sampio, Carratalá \& Valera-Ordaz, 2017) reveló que los encuadres mediáticos no solo resultaron hegemónicos en las informaciones periodísticas, sino que también fueron mayoritarios en las noticias que los propios partidos políticos publicaban en sus webs. Investigaciones similares sobre la misma campaña electoral (Vicente-Mariño \& Campos-Domínguez, 2017) coinciden en subrayar una cierta tendencia a impulsar mensajes encuadrados desde una lógica mediática incluso en las publicaciones que los partidos políticos realizaron en la red Facebook.

\subsection{De la calle a los partidos: la movilización por la independencia}

El debate en torno a la independencia de Cataluña respecto al estado español ha ido ganando peso en los últimos años en la esfera pública y en la escena política catalana hasta convertirse en el principal eje de confrontación y discusión partidista en la comunidad autónoma. La polarización del sistema político catalán en torno al soberanismo dio alguna señal inicial en las elecciones de 2000, aunque fundamentalmente se ha consolidado a partir de los comicios autonómicos celebrados en 2012, tras el pronunciamiento del Tribunal Constitucional que recortó significativamente el Estatuto de Autonomía de Cataluña, dando lugar a un escenario cada vez más plural, con la entrada de nuevas formaciones, pero también más polarizado, con el soberanismo como frontera divisoria (Medina, 2014). De este modo, las últimas convocatorias electorales que han tenido lugar en Cataluña han sido planteadas por los partidos políticos a partir de una lógica plebiscitaria, como ocurrió en 2015 (Martí \& Cetrà, 2016), cuando las dos principales formaciones independentistas pactaron una lista unitaria, lo que dio lugar a una campaña en la que los partidos apenas concretaron apuestas programáticas (López Meri, 2016) y en la que los medios de comunicación asumieron el marco de los comicios como plebiscito defendido por los partidos soberanistas (Ballesteros, 2017).

Sin embargo, aunque en los últimos años el debate independentista en Cataluña ha sido protagonizado por los partidos políticos, la cuestión soberanista nació y fue ganando apoyo ciudadano a partir de su articulación como acción colectiva. Desde sus inicios en 2009, la movilización en torno a la independencia ha estado ligada a la actividad de grupos de base y organizaciones de movimiento social, especialmente la Assemblea Nacional Catalana y Òmnium Cultural (Letamendia, 2017). En este sentido, esta expresión colectiva puede ser leída, en una primera etapa, como un movimiento popular que se desarrolla de abajo hacia arriba, que aprovecha la crisis socieconómica y política para articular un cuestionamiento a las instituciones. El surgimiento del movimiento independentista confluye de este modo con las demandas democráticas y de cambio social que, en paralelo, son promovidas por otros movimientos de acción ciudadana en contra del sistema (Clua i Fainé, 2014). Estas organizaciones, que no habían logrado mucha influencia antes de 2008, logran vehicular ilusiones y esperanzas apuntando a una mejora en las condiciones de vida de las personas si se consigue la independencia respecto a España y situando las ideas de libertad y dignidad como un logro épico al alcance del grupo (Sabucedo et al., 2017). El progresivo apoyo ganado por esta acción colectiva entre la sociedad catalana no se explica únicamente por cuestiones de mera identidad política. Como señalan algunos autores (Muñoz \& Tormos, 2015), el respaldo al soberanismo también tiene que ver con cuestiones como las expectativas económicas que se dibujan 
en un escenario de independencia, sobre todo entre aquellos que no están definidos identitariamente desde el punto de vista nacional o que no tienen una filiación política claramente ligada a un partido.

Como ocurre con el progresivo apoyo popular logrado por el independentismo, la cuestión de la identidad tampoco parece suficiente para explicar por sí sola la participación electoral en Catalunya. Investigaciones recientes (Fernández i Marín \& López, 2009, 2010) apuntan más bien a que la decisión de los ciudadanos catalanes de acudir o no a las urnas en las distintas convocatorias electorales depende sobre todo de un marco cultural de referencia, es decir, de filtros desde los que interpretar el mundo que superan la adscripción o identificación nacional y que se pueden situar a lo largo de un continuum entre una posición catalonocéntrica y otra hispanocéntrica. Estos marcos cognitivos estarían relacionados, entre otras variables, con el uso de la lengua y, también, con los medios de comunicación que son elegidos para informarse, una preferencia en la que el eje centro-periferia se revela como un predictor significativo (Valera-Ordaz, 2018).

El papel jugado por los medios de comunicación en el auge del independentismo en Cataluña ha sido objeto de diversos estudios recientes, sobre todo en torno al desarrollo de la consulta soberanista de noviembre de 2014 y las elecciones autonómicas de septiembre de 2015. Estas investigaciones (Ballesteros, 2015, 2017; Guillén \& Rodríguez-Díaz, 2017) han permitido subrayar el rol de los periódicos como actores políticos (Borrat, 1989) al demostrar que la prensa editada en Madrid y en Barcelona abordaron de forma distinta estos procesos electorales de acuerdo con sus respectivas líneas editoriales, como resulta coherente con la politización de los medios enmarcados en el modelo de pluralismo polarizado (Hallin \& Mancini, 2004). Los encuadres privilegiados por una y otra prensa, pese a ser diferentes, se corresponden con los propios de la lógica mediática (Guillén \& Rodríguez-Díaz, 2017) y orillan la cobertura de propuestas políticas, tal y como ocurrió con la comunicación en Twitter controlada por los propios partidos, que estuvo centrada en la cuestión soberanista y que apenas dio importancia a medidas programáticas (Meri, 2016).

Esta investigación tiene como propósito determinar si, de nuevo, fue la lógica mediática (encuadres estratégico y de juego) la que dominó la comunicación periodística y política durante la campaña electoral catalana de 2017, o si, en cambio, las excepcionales circunstancias de estos comicios (autonomía política suspendida y líderes ausentes de la campaña por encontrarse en prisión o huidos) privilegiaron encuadres propios de la filosofía política clásica (encuadre programático). O incluso, de la acción colectiva (encuadre prognosis), habida cuenta de la base social que ha permitido articular un progresivo apoyo ciudadano a la tesis independentista, y entendiendo que ese proyecto se corresponde con el escenario que todo movimiento social trata de dibujar como solución deseable a una situación de injusticia (Entman, 1993).

\section{Metodología}

El objetivo principal de esta investigación es identificar los encuadres hegemónicos en el discurso de los partidos políticos y en la cobertura de los medios de comunicación a lo largo de la campaña electoral catalana de 2017 (del 5 al 19 de diciembre).

La investigación compara los encuadres presentados por seis periódicos (El País, El Mundo, La Vanguardia, El Periódico de Catalunya, El Punt Avui y Ara) en sus titulares con los adoptados por los siete partidos políticos representados en el Parlament de Catalunya (JuntsXCat, ERC, PSC, PP, Ciudadanos, Catalunya en Comú-Podem y CUP) 
a través de las noticias publicadas en sus páginas web durante la campaña electoral catalana. Por tanto, la selección del corpus de análisis (Tabla 1) incluye:

- Informaciones publicadas por los siete partidos políticos con mayores posibilidades de representación en sus sitios web corporativos $(\mathrm{N}=150)$. Esta elección responde al hecho de que los partidos políticos dominan completamente la producción simbólica en sus webs (Dader, 2009) y, al hacerlo, eligen qué temas y encuadres privilegiar.

- Noticias de los principales periódicos de información general representativos de la prensa publicada en Madrid (El País y El Mundo) y en Barcelona ( $\mathrm{N}=494)$. En el último caso, se ha seleccionado una variedad de opciones que incluye tanto periódicos que no se posicionan a favor de la independencia (La Vanguardia y El Periódico de Catalunya) como también los que apoyan el programa independentista a través de su política editorial (El Punt Avui y Ara).

Tabla 1: Muestra analizada

\begin{tabular}{|c|c|c|c|}
\hline Partido Político & Unidades & Medio de comunicación & Noticias \\
\hline Ciudadanos (CS) & 22 & El País (EP) & 66 \\
\hline Junts per Catalunya (JxCat) & 27 & El Mundo (EM) & 55 \\
\hline $\begin{array}{l}\text { Esquerra Republicana de } \\
\text { Catalunya (ERC) }\end{array}$ & 7 & La Vanguardia (LV) & 94 \\
\hline $\begin{array}{l}\text { Partit dels Socialistes de } \\
\text { Catalunya (PSC) }\end{array}$ & 25 & $\begin{array}{l}\text { El Periódico de Catalunya } \\
\text { (EPC) }\end{array}$ & 93 \\
\hline $\begin{array}{l}\text { Catalunya en Comú-Podem } \\
\text { (CEPO) }\end{array}$ & 21 & El Punt-Avui (PA) & 93 \\
\hline $\begin{array}{l}\text { Candidatura de Unitat Popular } \\
\text { (CUP) }\end{array}$ & 23 & Ara (ARA) & 93 \\
\hline Partido Popular (PP) & 25 & & \\
\hline Total & 150 & Total & 494 \\
\hline
\end{tabular}

Fuente: Elaboración propia

La recogida de datos tuvo lugar del 5 al 19 de diciembre, en el caso de los sitios web de los partidos políticos. En el caso de los medios de comunicación examinados, el período de análisis comienza y finaliza el día posterior al de la jornada oficial de inicio y cierre de la campaña electoral. Esto es, del 6 al 20 de diciembre. La investigación trata de responder las siguientes preguntas de investigación:

PI 1: ¿Qué tipo de encuadre domina en las informaciones que ofrecen los partidos políticos a través de sus sitios web?

PI 2: ¿Qué tipo de encuadre prevalece en la cobertura que la prensa realizó de la campaña catalana de 2017 ?

PI 3: ¿Existen diferencias en los encuadres según los partidos políticos que protagonizan la información en función de la dialéctica constitucionalista-independentista?

PI 4: ¿Existen diferencias en los encuadres ofrecidos por los diferentes medios de acuerdo con sus políticas editoriales frente al proyecto independentista?

PI 5: ¿Qué encuadre tiene más peso en la primera semana en comparación con la segunda semana de campaña? 
El estudio se centra en los titulares de las noticias publicadas, una decisión contrastada en numerosas investigaciones sobre el empleo de encuadres periodísticos (Tankard, 2001; Trimble \& Sampert, 2004; Ballesteros, 2017; Palau et al., 2017) al considerar que los titulares son un atajo cognitivo de acceso a información más compleja (Andrew, 2007). La identificación del encuadre, en algunos casos más de uno, se basa en la revisión teórica sobre framing, tiene en cuenta cuatro categorías, aplicadas tanto a los medios de comunicación como a los comunicados de los partidos políticos (Tabla 2), y ya ha sido probada con éxito en investigaciones precedentes (Palau et al., 2017) que el presente estudio pretende replicar.

Tabla 2. Categorías de encuadre analizadas

\begin{tabular}{|ll|}
\hline Frame & Características \\
\hline Juego & $\begin{array}{l}\text { Referencias a ganadores/perdedores, a partir de encuestas, debates u otras expresiones } \\
\text { de la opinión pública; aprobación o rechazo por parte de grupos de interés, sectores o } \\
\text { personalidades de relevancia; especulaciones sobre potenciales pactos y coaliciones. }\end{array}$ \\
\hline Estrategia & $\begin{array}{l}\text { Relativo a las tácticas y movimientos empleados por los actores políticos para lograr } \\
\text { sus objetivos. Dada su complejidad se han diferenciado tres subestrategias: }\end{array}$ \\
& - Petición directa del voto o apelación al voto útil \\
- Estilos de liderazgo & - Ataque al rival político \\
Programático & Presentación de propuestas concretas recogidas en el programa electoral. \\
\hline prognosis & $\begin{array}{l}\text { Referencias al relato simbólico de los partidos sobre lo que está en juego en las } \\
\text { elecciones, y el plan de acción que conviene acometer. }\end{array}$
\end{tabular}

Fuente: Palau et al., 2017

\section{Resultados}

\subsection{Encuadres dominantes en las webs de los partidos políticos}

Las particularidades de la campaña, altamente influenciada por el debate en torno a la independencia de Cataluña, se reflejan en los comunicados de prensa ofrecidos por los diferentes partidos políticos. En primer lugar, cabe destacar que el encuadre más prominente en estas piezas es el de prognosis $(22,6 \%)$, lo que puede resultar bastante lógico si se tiene en cuenta que se trata de unos comicios en los que, según se desprende del discurso de los partidos, lo que está en juego es la identidad de la comunidad y el estatus legal del territorio. Por lo tanto, la hegemonía de esta dimensión encaja con las narrativas simbólicas que promueve el encuadre prognosis con respecto al diseño del modelo de país, como puede observarse en algunos de los titulares:

- Arrimadas: 'Quedan cuatro días para que en Cataluña haya un cambio de verdad y vuelvan los valores de libertad, igualdad, solidaridad y unión' (CS, 17/12/2017).

- Tenim ganes de tornar a aixecar aquest país i fer-lo gran [Tenemos ganas de volver a levantar el país y hacerlo grande] (PSC, 13/12/2017).

- President Puigdemont: "Volem fer un país millor entre tots $i$ aquí ens cal l'esperit de l'esport entès no com un element de competició, sinó de cohesió social" [Presidente Puigdemont: "Queremos hacer un país mejor entre todos y aquí necesitamos el espíritu 
deportivo entendido no como un elemento de competición, sino de cohesión social] (JxCat, 17/12/2017).

No obstante, la estrategia de ataque al oponente también evidencia una presencia importante en el conjunto de la muestra, ya que puede reconocerse en casi el $21 \%$ de los titulares analizados. Los siguientes dos casos lo ejemplifican claramente:

- El feixisme entra en campanya al Baix Montseny [El fascismo entra en campaña en el Baix Maestrat] (CUP, 9/12/2017).

- President Puigdemont a Iceta: "L’has feta tan grossa que la dimissió te la demanarà la vergonya d'haver empès els empresonaments i l'exili" [Presidente Puigdemont a Iceta: "La has liado de tal manera que la dimisión te la pedirá la vergüenza de haber empujado las detenciones y el exilio] (JxCat, 13/12/2017).

En segundo lugar, es importante enfatizar el escaso peso de los encuadres programático (propios de la lógica política) y de petición del voto (de carácter estratégico). Esto es particularmente sorprendente si se tiene en cuenta que son los partidos políticos los que proporcionan directamente la información en estas publicaciones electorales. Solo el $8,5 \%$ de las noticias publicadas en sus sitios web se corresponde con el encuadre de apelación al voto. La escasa presencia del encuadre programático resulta todavía más relevante si se considera que este período está oficialmente dedicado a la presentación de propuestas políticas: solo uno de cada 10 comunicados de prensa analizados trata sobre las iniciativas gubernamentales y las medidas específicas que proponen las formaciones.

Tabla 3. Tipos de encuadre en las informaciones de los partidos

\begin{tabular}{|l|r|r|}
\hline Encuadre & Número de piezas & $\%$ \\
\hline Juego & 39 & 22 \\
\hline Estratégico- petición de voto & 15 & 8,5 \\
\hline Estratégico- estilo de liderazgo & 28 & 15,8 \\
\hline Estratégico- ataque al oponente & 37 & 20,9 \\
\hline Programático & 18 & 10,2 \\
\hline prognosis & 40 & 22,6 \\
\hline Total & $177 *$ & 100 \\
\hline \multicolumn{2}{|c|}{$* 27$ de las unidades analizadas presentaban dos tipos de encuadre } \\
\hline
\end{tabular}

Fuente: Elaboración propia

Parece sustancial señalar que el encuadre de juego es el segundo más significativo (22\%). La presencia destacada de este encuadre muestra que la información de los partidos políticos reproduce los códigos y lógicas de los medios, como puede verse en los titulares que enfatizan la aprobación de ciertas formaciones por parte de grupos de interés o personalidades reconocidas:

- L'escriptor britànic Owen Jones dóna suport a Catalunya en Comú - Podem per "acabar amb l'imperi de les elits" [El escritor británico Owen Jones apoya a Catalunya en Comú - Podem para "acabar con el imperio de las élites"] (CEPO, 15/12/2017).

- Joves talents i perfils consolidats, entre els suports a la candidatura d'Esquerra [Jóvenes talentos y perfiles consolidados, entre los apoyos a la candidatura de Esquerra] (ERC, 19/12/2017). 
Los titulares que responden a esta categoría también ofrecen proyecciones vinculadas a resultados hipotéticos y a los diferentes roles que las distintas fuerzas políticas podrían desempeñar en las negociaciones parlamentarias para formar gobierno:

- Inés Arrimadas: "Ciudadanos es la única llave que puede abrir una nueva etapa política de convivencia en Cataluña” (CS, 8/12/2017).

- García Albiol "un gobierno constitucionalista pasa necesariamente por un PP fuerte en el Parlament de Cataluña” (PP, 19/12/2017).

\subsection{Encuadres dominantes en la cobertura periodística}

Las noticias analizadas permiten observar la extraordinaria relevancia $(29,3 \%)$ que la prensa otorga al encuadre estratégico relacionado con el estilo de liderazgo en los titulares vinculados con la campaña electoral catalana, superando incluso los que responden al encuadre de juego (26,3\%), que generalmente es el más prominente en la cobertura mediática, por la tendencia de los medios de comunicación a presentar las elecciones como si de una especie de competencia o carrera se tratara. La singularidad de esta campaña electoral podría explicar la notable relevancia de los titulares que resaltan las estrategias políticas (los tres sub-encuadres considerados bajo esta categoría suponen en total el $47 \%$ de la muestra), en particular, como dijimos, los estilos de liderazgo representados por los diferentes oponentes políticos:

- Rajoy avisa que volverá a aplicar el artículo 155 si lo ve necesario (LV, 18/12/2017).

- Domènech promete "recoser" en lugar de "descabezar y desinfectar" (LV, 18/12/2017).

- Junqueras: "Soc a la presó perquè no m'amago i soc conseqüent" [Junqueras: "Estoy en prisión porque no me escondo y soy consecuente"] (ARA, 19/12/2017).

- Arrimadas modera el seu discurs per distanciar-se del PP [Arrimadas modera su discurso para distanciarse del PP] (ARA, 9/12/2017).

El sub-encuadre de ataque al oponente incluido en la categoría estratégica es especialmente feroz, sobre todo en aquellos titulares que confrontan a representantes de partidos pro independencia con líderes constitucionalistas, como puede verse en los siguientes ejemplos: "Albiol acusa a Puigdemont de actuar como un dictador" (LV, 16/12/2017) o "La CUP invoca l'esperit de l'1-O per vèncer el feixisme, en lloc d'adormir-lo" ["La CUP invoca al espíritu del 1-O para vencer al fascismo en lugar de adormecerlo"] (PA, 06/12/2017). Ambos titulares, irónicamente de los partidos políticos situados en los extremos opuestos del espectro político, recurren a los mismos argumentos para atacar a los respectivos rivales políticos.

En contraste con la relevancia del encuadre estratégico, poco más de una cuarta parte de los titulares $(26,3 \%)$ se corresponden con la categoría de juego. Se trata mayoritariamente de noticias que subrayan los movimientos políticos de las formaciones concurrentes con el fin de formar un hipotético gobierno tras la celebración de las elecciones: "El plan de Cs: un Govern con Iceta e independientes" (EM, 7/12/2017) o "Podemos baraja como única opción otro tripartito" (EP, 9/12/2017).

Finalmente, el 16,8\% de los titulares de la muestra se centran en cuestiones programáticas $(7,2 \%)$ o se corresponden con una dimensión prognóstica, de mayor contenido simbólico $(9,6 \%)$. La presencia de estos encuadres en la prensa se encuentra por debajo de la mitad del peso que manifiestan en los comunicados de prensa de los partidos políticos, lo que 
muestra claramente el interés de los medios por priorizar en sus informaciones problemas de competencia o rivalidad entre las formaciones políticas en liza.

Tabla 4. Tipos de encuadre en las informaciones de la prensa

\begin{tabular}{|l|r|r|}
\hline Encuadre & Número de piezas & $\%$ \\
\hline Juego & 132 & 26,3 \\
\hline Estratégico- petición de voto & 56 & 11,2 \\
\hline Estratégico- estilo de liderazgo & 147 & 29,3 \\
\hline Estratégico- ataque al oponente & 82 & 16,4 \\
\hline Programático & 36 & 7,2 \\
\hline prognosis & 48 & 9,6 \\
\hline Total & $501 *$ & 100 \\
\hline *Siete de las unidades analizadas presentaban dos tipos de encuadre
\end{tabular}

Fuente: Elaboración propia

No obstante, es posible observar importantes diferencias al comparar la cobertura de los distintos partidos políticos en los medios de comunicación analizados. En este sentido, puede verse que no solo el encuadre más relevante, sino también su representatividad, difiere dependiendo de la organización política que protagoniza la noticia. El encuadre de juego alcanza un $37,7 \%$ de los titulares dedicados a la cobertura de Catalunya en ComúPodem, pero es casi veinte puntos porcentuales menos importante en el caso de ERC $(18,2 \%)$. El encuadre estratégico vinculado al estilo de liderazgo es el más destacado en cuatro de cada diez noticias relacionadas con JxCat (41\%) y ERC (43\%), pero reduce su representatividad hasta un $11,3 \%$ en el caso de las noticias vinculadas con la CUP. Esta última formación destaca por ser caracterizada como la que privilegia el ataque al oponente, encuadre estratégico que puede observarse priorizado en ejemplos como "Gabriel retreu a Santamaría: "On és la divisó de poders?"” [Gabriel reprocha a Santamaría: “¿Dónde está la división de poderes?”] (PA, 18/12/2017), “La CUP titlla de "feixistes" $i$ "terroristes" el bloc del 155" ["La CUP tilda de 'fascistas' y 'terroristas' al bloque del 155"] (ARA, 17/12/2017) o "La CUP arremete contra el PSC" (EM, 20/12/2017). Por otro lado, el encuadre de estrategia vinculado a la petición del voto es el hegemónico en las noticias sobre Ciudadanos (22,7\%), pero es prácticamente irrelevante en el caso del partido de la CUP $(3,77 \%)$.

Los encuadres programático y prognosis también presentan una variación significativa al considerar a los diferentes partidos políticos. El frame sobre medidas y programa es el más relevante en las informaciones dedicadas a Catalunya en Comú-Podem, al observarse en el 29\% de los titulares, mientras que representa un escaso $3 \%$ en el caso de Ciudadanos. Por el contrario, CUP ocupa el primer lugar si se centra la atención en la presencia del encuadre prognosis en la cobertura de la campaña (17\%), mientras que PSC, ERC y JxCat no superan el $6 \%$ en las noticias categorizadas bajo esta dimensión. 
Tabla 5. Tipos de encuadre en las informaciones de la prensa en función del partido político

\begin{tabular}{|c|c|c|c|c|c|c|c|c|c|c|c|c|c|}
\hline & \multicolumn{2}{|c|}{$\begin{array}{l}\text { Encuadre } \\
\text { de juego }\end{array}$} & \multicolumn{2}{|c|}{$\begin{array}{l}\text { Encuadre de } \\
\text { estrategia - } \\
\text { apelar voto }\end{array}$} & \multicolumn{2}{|c|}{$\begin{array}{c}\text { Encuadre de } \\
\text { estrategia - } \\
\text { liderazgo }\end{array}$} & \multicolumn{2}{|c|}{$\begin{array}{l}\text { Encuadre de } \\
\text { estrategia - } \\
\text { ataque rival }\end{array}$} & \multicolumn{2}{|c|}{$\begin{array}{c}\text { Encuadre } \\
\text { programático }\end{array}$} & \multicolumn{2}{|c|}{$\begin{array}{l}\text { Encuadre } \\
\text { prognosis }\end{array}$} & \multirow{2}{*}{$\begin{array}{l}\text { Total } \\
\text { N }\end{array}$} \\
\hline & $\mathrm{N}$ & $\%$ & $\mathrm{~N}$ & $\%$ & $\mathrm{~N}$ & $\%$ & $\mathrm{~N}$ & $\%$ & $\mathrm{~N}$ & $\%$ & $\mathrm{~N}$ & $\%$ & \\
\hline CS & 23 & 30,7 & 17 & 22,66 & 19 & 25,33 & 6 & 8 & 2 & 2,66 & 8 & 10,66 & 75 \\
\hline PSC & 20 & 24,7 & 9 & 11,1 & 21 & 25,92 & 12 & 14,81 & 14 & 17,28 & 5 & 6,17 & 81 \\
\hline $\begin{array}{c}\text { CEP } \\
\text { O }\end{array}$ & 26 & 37,7 & 7 & 10,14 & 11 & 15,94 & 15 & 21,73 & 2 & 28,98 & 8 & 11,59 & 69 \\
\hline PP & 13 & 19,1 & 6 & 8,82 & 25 & 36,76 & 13 & 19,11 & 3 & 4,11 & 8 & 11,76 & 68 \\
\hline JxCat & 18 & 23,1 & 9 & 11,53 & 32 & 41,02 & 10 & 12,82 & 4 & 5,12 & 5 & 6,41 & 78 \\
\hline ERC & 14 & 18,2 & 6 & 7,79 & 33 & 42,85 & 11 & 14,28 & 8 & 10,38 & 5 & 6,49 & 77 \\
\hline CUP & 18 & 33,9 & 2 & 3,8 & 6 & 11,3 & 15 & 28,3 & 3 & 5,66 & 9 & 17 & 53 \\
\hline & 132 & & 56 & & 147 & & 82 & & 36 & & 48 & & 501 \\
\hline
\end{tabular}

Fuente: Elaboración propia

\subsection{Encuadres en prensa de acuerdo a la dialéctica constitucionalista- independentista}

Uno de los aspectos más interesantes de analizar en la campaña catalana es el enfrentamiento entre los dos bloques representados por los partidos pro independencia (IND), por un lado, y por las formaciones unionistas, aquellas que apoyan al Estado autonómico regulado por la Constitución española (constitucionalistas, CONS), por otro. Se ha incluido en el primer bloque a los tres partidos políticos que apoyaron la declaración de independencia de Cataluña en el Parlament catalán el 27 de octubre de 2017: Junts per Catalunya (JxCat), Esquerra Republicana de Catalunya (ERC) y Candidatura de Unitat Popular (CUP). Por otro lado, se han identificado como constitucionalistas las cuatro fuerzas políticas que se mostraron contrarias a esta declaración: Ciudadanos (CS), Partit dels Socialistes de Catalunya (PSC), Partido Popular (PP) y Catalunya en Comú-Podem (CEPO).

El primer aspecto relevante es que las mayores diferencias entre las dos tendencias políticas se concentran en tres tipos de encuadres: programático, estrategia-estilo de liderazgo y juego. Por un lado, las noticias dedicadas a cubrir la actividad de los partidos unionistas son las que presentan con mayor frecuencia titulares de encuadre de juego (26\%), cinco puntos más que aquellas piezas que cubren la actualidad de las formaciones independentistas. Las informaciones sobre los partidos constitucionalistas también destacan por hacer un uso regular del encuadre estratégico de estilos de liderazgo $(18,3 \%)$, seis puntos por encima de las noticias protagonizadas por las fuerzas políticas que defienden la independencia de Cataluña. Por otro lado, el encuadre programático es más habitual en las piezas relacionadas con las fuerzas políticas a favor de la independencia $(16,1 \%)$, superando más del doble de la frecuencia con la que esta dimensión sobre medidas concretas es priorizada en las noticias sobre partidos en contra del secesionismo. 
Tabla 6. Tipos de encuadre en las informaciones de la prensa en función de la dialéctica constitucionalista-independentista

\begin{tabular}{|c|c|c|c|c|c|c|c|c|c|c|c|c|c|}
\hline & \multicolumn{2}{|c|}{$\begin{array}{l}\text { Encuadre } \\
\text { de juego }\end{array}$} & \multicolumn{2}{|c|}{$\begin{array}{l}\text { Encuadre de } \\
\text { estrategia - } \\
\text { apelar voto }\end{array}$} & \multicolumn{2}{|c|}{$\begin{array}{l}\text { Encuadre de } \\
\text { estrategia - } \\
\text { liderazgo }\end{array}$} & \multicolumn{2}{|c|}{$\begin{array}{l}\text { Encuadre de } \\
\text { estrategia - } \\
\text { ataque rival }\end{array}$} & \multicolumn{2}{|c|}{$\begin{array}{c}\text { Encuadre } \\
\text { programático }\end{array}$} & \multicolumn{2}{|c|}{$\begin{array}{l}\text { Encuadre } \\
\text { prognosis }\end{array}$} & \multirow[t]{2}{*}{ Total } \\
\hline & $\mathrm{N}$ & $\%$ & $\mathrm{~N}$ & $\%$ & $\mathrm{~N}$ & $\%$ & $\mathrm{~N}$ & $\%$ & $\mathrm{~N}$ & $\%$ & $\mathrm{~N}$ & $\%$ & \\
\hline CONS & 26 & 26 & 9 & 7,8 & 21 & 18,3 & 25 & 21,7 & 8 & 7 & 26 & 22,6 & 115 \\
\hline IND & 13 & 21 & 6 & 9,7 & 7 & 11,3 & 12 & 19,3 & 10 & 16,1 & 14 & 22,6 & 62 \\
\hline
\end{tabular}

Fuente: Elaboración propia

\subsection{Líneas editoriales y encuadres de cobertura en la dialéctica constitucionalista- independentista}

La muestra analizada incluye todos los partidos políticos representados en el Parlament catalán, pero también periódicos que muestran una variedad de políticas editoriales, lo que permite comparar diferentes enfoques en la cobertura electoral. Teniendo en cuenta los medios analizados, se puede distinguir entre los periódicos editados en Madrid (prensa de Madrid), otros editados en Barcelona pero que no defienden una posición independentista (prensa de Barcelona) y otros dos periódicos producidos desde Barcelona que sí apoyan abiertamente el proceso de independencia (prensa pro independencia).

Tabla 7. Líneas editoriales y cobertura de partidos constitucionalistas e independentistas

\begin{tabular}{|c|c|c|c|c|c|c|c|c|c|c|c|c|c|c|}
\hline & & \multicolumn{2}{|c|}{$\begin{array}{l}\text { Encuadre } \\
\text { de juego }\end{array}$} & \multicolumn{2}{|c|}{$\begin{array}{l}\text { Encuadre de } \\
\text { estrategia - } \\
\text { apelar voto }\end{array}$} & \multicolumn{2}{|c|}{$\begin{array}{l}\text { Encuadre de } \\
\text { estrategia - } \\
\text { liderazgo }\end{array}$} & \multicolumn{2}{|c|}{$\begin{array}{l}\text { Encuadre de } \\
\text { estrategia - } \\
\text { ataque rival }\end{array}$} & \multicolumn{2}{|c|}{$\begin{array}{c}\text { Encuadre } \\
\text { programático }\end{array}$} & \multicolumn{2}{|c|}{$\begin{array}{l}\text { Encuadre } \\
\text { prognosis }\end{array}$} & \multirow[t]{2}{*}{ Total } \\
\hline & & $\mathrm{N}$ & $\%$ & $\mathrm{~N}$ & $\%$ & $\mathrm{~N}$ & $\%$ & $\mathrm{~N}$ & $\%$ & $\mathrm{~N}$ & $\%$ & $\mathrm{~N}$ & $\%$ & \\
\hline \multirow{2}{*}{$\begin{array}{l}\text { Prensa } \\
\text { Pro } \\
\text { indepen }\end{array}$} & CONS & 23 & 21,1 & 14 & 12,8 & 33 & 30,3 & 19 & 17,5 & 9 & 8,2 & 11 & 10,1 & 109 \\
\hline & IND & 20 & 25 & 8 & 10 & 29 & 36,2 & 13 & 16,3 & 4 & 5 & 6 & 7,5 & 80 \\
\hline \multirow{2}{*}{$\begin{array}{l}\text { Prensa } \\
\text { de } B C N\end{array}$} & CONS & 31 & 26,3 & 14 & 11,8 & 28 & 23,7 & 20 & 16,9 & 9 & 7,6 & 16 & 13,5 & 118 \\
\hline & IND & 19 & 26,7 & 8 & 11,3 & 17 & 23,9 & 10 & 14,1 & 6 & 8,4 & 11 & 15,5 & 71 \\
\hline \multirow{2}{*}{$\begin{array}{l}\text { Prensa } \\
\text { Madrid }\end{array}$} & CONS & 30 & 42,2 & 11 & 15,5 & 16 & 22,5 & 8 & 11,3 & 3 & 4,2 & 3 & 4,2 & 71 \\
\hline & IND & 9 & 17,3 & 1 & 1,9 & 24 & 46,1 & 12 & 23,1 & 5 & 9,6 & 1 & 1,9 & 52 \\
\hline Total & 109 & & 80 & & 118 & & 71 & & 71 & & 52 & & & \\
\hline
\end{tabular}

\section{Fuente: Elaboración propia}

Los resultados de la investigación muestran diferencias relevantes al observar la cobertura de los periódicos publicados en Madrid, en particular relacionadas con el empleo del encuadre estratégico de estilo de liderazgo y el de juego. En ambos casos, los titulares de EM y EP presentan más de 20 puntos porcentuales de diferencia respecto a la utilización de estos encuadres en ambos bloques políticos: los partidos constitucionalistas son los que, con mayor frecuencia, son objeto de cobertura desde el encuadre de juego (42,25\%), mientras que en las noticias sobre las formaciones independentistas destaca el encuadre estratégico de estilo de liderazgo (46,15\%). Si se observa la prensa publicada en Madrid, los partidos unionistas tienden a centrarse más en la solicitud de voto $(15,5 \%)$ ("Rajoy: 'El voto al PP es el único no sujeto a cambalaches'”, EM, 09/12/2017) que los independentistas $(1,9 \%)$, mientras que las noticias sobre estos últimos emplean con mayor 
frecuencia el encuadre estratégico de ataque al rival $(23,1 \%)$ ("Forcadell radicaliza su discurso y eleva la amenaza a la Justicia”, EM, 17/12/2017) que las informaciones protagonizadas por formaciones políticas de perfil constitucionalista.

El estudio también subraya que los dos periódicos considerados como representantes de la prensa barcelonesa que no apoya el proyecto independentista ( $L a$ Vanguardia y El Periódico de Cataluña) proporcionan el enfoque más equilibrado de los dos bloques políticos en confrontación. Es decir, con una representación casi equitativa de los diferentes encuadres considerados en la cobertura del bloque constitucionalista y en la del bloque independentista. Su tratamiento informativo no privilegia encuadres distintos dependiendo de la posición de las formaciones políticas ante el planteamiento soberanista. Los periódicos Ara y El Punt Avui, categorizados como prensa independentista, sí difieren, en cambio, en cuanto a los encuadres favorecidos en cada uno de los dos bloques políticos. Sin embargo, esas diferencias son menos relevantes que las observadas en la prensa editada en Madrid y se concentran principalmente en dos encuadres: el de juego y el de estilo de liderazgo, algo más frecuentes en las noticias dedicadas a cubrir la actividad de partidos soberanistas que en las informaciones sobre los unionistas. Cabe destacar, por último, que mientras la prensa publicada en Madrid tiende a resaltar el encuadre de juego al abordar los partidos constitucionalistas, la prensa independentista hace lo mismo con los partidos que comulgan con su línea editorial ( $25 \%$ contra un $21,1 \%$ ), pero coinciden con la cobertura de la prensa de Madrid si se observa el peso del encuadre de estilo de liderazgo en los partidos independentistas $(36,2 \%)$ sobre los constitucionalistas $(30,3 \%)$, aunque la diferencia de seis puntos sea menor a la observada en los periódicos publicados desde la capital del estado $(46,1 \%$ frente a $22,5 \%)$.

\subsection{Evolución de los encuadres a lo largo de la campaña}

Además del discurso de los partidos políticos y de las diferencias en la cobertura de los medios, la naturaleza de la campaña política está claramente marcada por el día de las elecciones, que puede considerarse como un punto de referencia en el análisis. De acuerdo con esto, se han dividido las dos semanas de campaña para ver si la proximidad del día de la votación influye en la priorización de algunos encuadres sobre otros, tanto en el caso de los sitios web de información de los partidos políticos como también en los titulares publicados por los periódicos objeto de estudio. Los resultados de la investigación muestran diferencias relevantes en la evolución del encuadre del juego. Los medios periodísticos duplicaron la presencia de este en la segunda semana (de observarse en el $18,1 \%$ de las piezas pasó a ser identificado en el 35,4\% de las unidades), mientras que también aumentó cuatro puntos porcentuales en los comunicados de los partidos políticos (del 20,4 al 24\%). Por el contrario, encuadres como el estratégico de estilo de liderazgo o del ataque al oponente tendieron a ser menos prominentes a medida que avanza la campaña, especialmente si se observa la cobertura de la prensa.

En contraste con lo que podría esperarse, el encuadre programático perdió peso de la primera (del 6 al 13 de diciembre) a la segunda semana (del 14 al 20 de diciembre) tanto en los medios de comunicación como, sobre todo, en los sitios web de los partidos políticos. El encuadre de prognosis, por el contrario, muestra una dinámica menos regular, pues mientras disminuyó en la cobertura de los medios de comunicación (del 11,85\% al $7,2 \%$ ) ganó fuerza en las publicaciones de los partidos políticos (del 21,4\% al 24\%).

Tabla 8. Evolución de los encuadres a lo largo de la campaña 


\begin{tabular}{|l|c|c|c|c|c|c|c|c|}
\hline & \multicolumn{4}{|c|}{ Prensa } & \multicolumn{4}{c|}{ Webs partidos políticos } \\
\hline & \multicolumn{2}{|c|}{$\mathbf{1}^{\mathbf{a}} \mathbf{s e m a n a}$} & \multicolumn{2}{|c|}{$\mathbf{2}^{\mathbf{a}} \mathbf{s e m a n a}$} & \multicolumn{2}{|c|}{$\mathbf{1}^{\mathbf{a}}$ semana } & $\mathbf{2}^{\mathbf{a}}$ semana \\
\hline Encuadre & $\mathbf{N}$ & $\mathbf{\%}$ & $\mathbf{N}$ & $\mathbf{\%}$ & $\mathbf{N}$ & $\mathbf{\%}$ & $\mathbf{N}$ & $\mathbf{\%}$ \\
\hline Juego & 48 & 18,1 & 84 & 35,4 & 20 & 20,4 & 19 & 24 \\
\hline Estratégico- petición de voto & 29 & 13 & 27 & 11,4 & 7 & 7,1 & 8 & 10,2 \\
\hline Estratégico- estilo de liderazgo & 87 & 33 & 60 & 25,3 & 15 & 15,3 & 13 & 16,4 \\
\hline Estratégico- ataque al oponente & 48 & 18,1 & 34 & 14,4 & 23 & 23,5 & 14 & 17,7 \\
\hline Programático & 21 & 8 & 15 & 6,3 & 12 & 12,3 & 6 & 7,6 \\
\hline prognosis & 31 & 11,8 & 17 & 7,2 & 21 & 21,4 & 19 & 24 \\
\hline Total & 264 & & 237 & & 98 & & 79 \\
\hline
\end{tabular}

Fuente: Elaboración propia

\section{Discusión y conclusiones}

Los resultados de la investigación subrayan el predominio del encuadre de juego y del estratégico ligado al estilo de liderazgo, en sintonía con lo que ya se ha comprobado en estudios anteriores sobre la cobertura de la campaña electoral (Palau-Sampio et al., 2017), por delante del encuadre programático, tanto en la información proporcionada por los partidos políticos como en la publicada por los medios de comunicación. Sin embargo, el carácter excepcional de estas elecciones, celebradas después de una declaración de independencia, con la autonomía regional suspendida por el gobierno central, con un candidato cabeza de lista en prisión y otro en Bruselas, reclamado por los tribunales, ha condicionado algunos aspectos importantes.

En primer lugar, debe destacarse la importancia del encuadre de prognosis, particularmente en la información producida por los partidos políticos, en la que coincide en relevancia junto con el encuadre de juego. La presencia del encuadre propio de movilización en la producción comunicativa de las organizaciones políticas es un elemento significativo y diferencial de este estudio de caso si lo comparamos con análisis anteriores sobre la comunicación política en las campañas electorales. Su prevalencia en la campaña analizada puede vincularse con el carácter fuertemente simbólico de estas elecciones, que muchos partidos enfrentaron como una oportunidad histórica de construir un nuevo país, independiente o no, y también con el hecho de que el movimiento soberanista se articulara inicialmente como acción colectiva, desde la dinámica de las organizaciones sociales, y nutrido por tanto de un tipo de narrativa de carácter emotivo y esperanzador que quizá los partidos políticos pro independencia han querido adoptar para tratar de activar electoralmente el mayor número posible de ciudadanos.

De este modo, los partidos políticos no enfatizan medidas o propuestas específicas, sino ideas evocadoras con la capacidad de resonar en las mentes de los individuos. Este encuadre de prognosis también gana presencia a medida que avanza la campaña. En este sentido, los mensajes de las organizaciones políticas subrayan los valores y las emociones en sus publicaciones, acercándose al discurso de las organizaciones sociales, que también había sido esencial y exitoso en la activación del movimiento pro independencia en los últimos años, especialmente de la mano de la ANC y Òmnium Cultural. Así, no es extraño que los partidos independentistas hayan acercado su mensaje a esa lógica prognóstica. Este encuadre es, sin embargo, mucho menor en las noticias sobre la campaña publicadas 
en prensa, que se alejan de un discurso de acción colectiva que desafía significativamente la lógica de los medios. El predominio del encuadre de prognosis en el discurso de los partidos se ve reforzado por una presencia notable de mensajes que se presentan como un ataque al oponente, de tal manera que la delimitación de los grupos nosotros/ellos, necesaria en cualquier acción de movilización colectiva, también se logra con éxito.

En segundo lugar, la importancia del encuadre estratégico de estilo de liderazgo en la cobertura de los periódicos puede relacionarse con la particularidad de estas elecciones, en las que los líderes de dos partidos pro independencia estuvieron ausentes durante la campaña. Además, el estilo de liderazgo se puede leer como una metáfora del estilo de país que quieren construir. Este enfoque se ve reforzado por la creciente tendencia a la personalización de la información política (Echevarría, 2017). Líderes -en situaciones inusuales- eclipsan a la organización política. Tanto Carles Puigdemont como Oriol Junqueras se convierten en la cara del programa pro independencia y, a pesar de no estar físicamente en Cataluña, sus nombres propios impregnan de forma mucho más clara el discurso informativo que las siglas de sus organizaciones políticas. Sin embargo, los resultados apuntan a que la CUP no se vio afectada por esta tendencia: su estructura, escasamente jerárquica, su carácter asambleario y un líder sin demasiada prominencia en la fase anterior de conflicto, hacen que resulte difícil a los medios centrar su cobertura en una sola cara de la organización. Por su parte, el caso de la candidata de Ciudadanos, Inés Arrimadas, también atrae la atención de los medios por razones obvias: por primera vez, una mujer como candidata de un partido no nacionalista puede ganar las elecciones según las encuestas. Es el contrapunto perfecto para los líderes independentistas.

La alta presencia del encuadre de juego en ambas muestras (unidades periodísticas y mensajes producidos por los partidos) resalta, en tercer lugar, la preponderancia de las referencias a los hipotéticos pactos y apoyos después de la votación, así como de la dinámica de ganar/perder como trasfondo de la información de campaña. Es importante tener en cuenta que también las noticias que aparecen en los sitios web de los partidos suelen recurrir a este encuadre, tradicionalmente considerado más típico de la dinámica de la cobertura de los medios y capaz de aumentar el interés de los ciudadanos en el seguimiento de la campaña como competición (Iyengar, Norpoth \& Hahn, 2004). Tanto en el caso de la información producida por los partidos políticos como en las noticias, puede verse que este encuadre es especialmente importante en mensajes relacionados con organizaciones como Catalunya En Comú-Podem o Ciudadanos. Es decir, el encuadre de juego es claramente significativo en la llamada "nueva política". Estas organizaciones, que han crecido en gran medida gracias a su elevada presencia en programas de televisión, muestran una mayor lógica mediática en su producción discursiva. El encuadre de juego también es más frecuente en el conjunto de fuerzas constitucionalistas que en los partidos independentistas, que, en cambio, ponen mayor esfuerzo en resaltar temas programáticos específicos, probablemente con el objetivo de aportar cierta seriedad a su propuesta de construir un estado diferente al de España y reducir la incertidumbre. En cualquier caso, el encuadre de juego es clave tanto en mensajes periodísticos como políticos, a pesar de su carácter especulativo, ganando presencia a medida que avanza la campaña.

Respecto a los enfoques de los periódicos considerando sus políticas editoriales, puede observarse, en cuarto lugar, que la prensa catalana no revela contrastes significativos entre el tratamiento a los distintos partidos políticos durante la cobertura de la campaña a pesar de su proximidad física y emocional a los hechos cubiertos. Este no es el caso, sin embargo, de la prensa producida en Madrid. Tanto El País como El Mundo ofrecen un enfoque notablemente maniqueo, dado el trato diferente otorgado a los partidos constitucionalistas y a los partidos a favor de la independencia. Estos periódicos, dirigidos 
principalmente a una audiencia bastante alejada de la realidad catalana y que no votará en las elecciones, representan a los partidos pro independencia a partir de los encuadres de estrategia ligados al estilo de liderazgo y al ataque al adversario, lo que facilita presentar a las organizaciones independentistas a partir de una aproximación centrada en sus líderes, caracterizados como actores violentos y amenazadores. El impacto de este enfoque, que debería analizarse más a fondo en futuros estudios, podría interpretarse en términos de política estatal y en base a la lógica electoral de los partidos españoles.

En conclusión, a pesar de las condiciones excepcionales que rodearon esta campaña electoral, los hallazgos revelan la adopción de la lógica de los medios por parte de los partidos políticos, ya que puede identificarse un alto grado de encuadres de estrategia y de juego en los mensajes publicados por ambos actores. Aunque la exposición de medidas políticas específicas es escasa, es importante señalar que el contexto político particular contribuye a una mayor presencia del encuadre de prognosis en comparación con otros procesos electorales examinados en estudios precedentes, vinculado especialmente a las proyecciones simbólicas y utópicas presentadas por los partidos pro independencia y al escenario de desastre propuesto por los unionistas. Por lo tanto, en este proceso electoral único, los partidos políticos muestran un claro interés por movilizar a los ciudadanos a través de discursos cercanos a la lógica de acción colectiva.

\section{Bibliografía}

Aalberg, T., Strömback, J., \& De Vreese, C. H. (2011). The framing of politics as strategy and game: a review of concepts, operationalizations and key findings. Journalism, 13(2), 162-178. https://doi.org/10.1177/1464884911427799

Andrew, B. C. (2007). Media-generated shortcuts: Do newspaper headlines present another roadblock for low information rationality? The International Journal of Press/Politics, 12(2), 24-43. https://doi.org/10.1177/1081180X07299795

Ballesteros Herencia, C. A. (2015). Naciones mediáticas. Los marcos informativos de la consulta sobre independencia de Cataluña (9-N). Doxa Comunicación, 20, 161-183.

Ballesteros Herencia, C. A. (2017). Siguiendo al flautista. Framing del independentismo catalán en las votaciones de 2014 y 2016. Estudios sobre el mensaje periodístico, 23(1), 307-323. http://dx.doi.org/10.5209/ESMP.55598

Barreiro Rivas, X. L., Pereira López, M., \& García Hípola, G. (2015). Los efectos sobre el voto de la campaña electoral en las elecciones europeas de 2014 en España. Revista Española de Ciencia Política, 39, 67-93.

Berganza, R. (2008). Medios de comunicación, "espiral del cinismo" y desconfianza política. Estudio de caso de la cobertura mediática de los comicios electorales europeos. Zer: Revista de estudios de comunicación, 13(25), 121-139.

Berrocal, S., \& Fernández, C. (2006). Las elecciones legislativas de 2004. Un análisis de las encuestas y de la gestión comunicativa en la campaña electoral: su proyección en la decisión de voto. Doxa Comunicación, 4, 189-208.

Blumenthal, S. (1980). The permanent campaign: Inside the world of elite political operations. Boston: Beacon Press. 
Blumler, J. G., \& Esser, F. (2018). Mediatization as a combination of push and pull forces: Examples during the 2015 UK general election campaign. Journalism. https://doi.org/10.1177/1464884918754850

Borrat, H. (1989). El periódico, actor político. Barcelona: Gustavo Gili.

Cappella, J. N., \& Jamieson, K. H. (1996). News frames, political cynicism, and media cynicism. The annals of the American Academy of Political and Social Science, 546, 7184. https://doi.org/10.1177/0002716296546001007

Clua i Fainé, M. (2014). Identidad y política en Cataluña: el auge del independentismo en el nacionalismo catalán actual. Quaderns-e de l'Institut Català d'Antropologia, 19(2), 7999.

Dader, J. L. (2009). Ciberpolítica en los websites de partidos políticos: la experiencia de las elecciones de 2008 en España ante las tendencias transnacionales. Revista de sociología y política, 17(34), 45-62. $\quad$ http://dx.doi.org/10.1590/S0104$\underline{44782009000300005}$

Davis, A. (2007). Investigating Journalist Influences on Political Issue Agendas at Westminster. Political Communication, 24(2), 181-199. https://doi.org/10.1080/10584600701313033

Dayan, D., \& Katz, E. (1992). Media events: The live broadcasting of history. Cambridge, MA: Harvard University Press.

De-Vreese, C. H. (2005). The Spiral of Cynicism Reconsidered. European Journal of Communication, 20(3), 283-301. https://doi.org/10.1177/0267323105055259

De Vreese, C. H., \& Semetko, H. (2002). Cynical and Engaged: Strategic Campaign Coverage, Public Opinion, and Mobilization in a Referendum. Communication Research, 29(6), 615-641. https://doi.org/10.1177/009365002237829

Echeverría, M. (2017). Personalización política e infoentretenimiento periodístico. Un estudio desde los encuadres. Cuadernos.info, 41, 71-87. https://doi.org/10.7764/cdi.41.1099

Entman, R. (1993). Framing: Toward Clarification of a Fractured Paradigm. Journal of Communication, 43(4), 51-58. https://doi.org/10.1111/j.1460-2466.1993.tb01304.x

Farrell, D., \& Schmitt-Beck, R. (Eds.). (2002). Do political campaigns matter?: campaign effects in elections and referendums. London: Routledge.

Fernández i Marín, X., \& López, J. (2009). Abstenció a Catalunya: una qüestió de marc cognitiu. Eines per a l'esquerra nacional, 10, 23-38.

Fernández i Marín, X., \& López, J. (2010). Marco cultural de referencia y participación electoral en Cataluña. Revista Española de Ciencia Política, 23, 31-37.

Guillen, A., \& Rodríguez-Diaz, R. (2017). Frames y agendas durante el proceso soberanista catalán (2013-2015). Sur le journalisme, 6(2), 140-157.

Hallin, D., \& Mancini, P. (2004). Comparing Media Systems: Three Models of Media and Politics. Cambridge: Cambridge University Press.

Iyengar, S. (1991). Is Anyone Responsible? How Television Frames Political Issues. Chicago: The University of Chicago Press. 
Iyengar, S., Norpoth, H., \& Hahn, K. S. (2004). Consumer Demand for Election News: The Horserace Sells. Journal of politics, 66(1), 157-175. https://doi.org/10.1046/j.14682508.2004.00146.x

Letamendia, A (2018). Movilización, represión y voto: rastreando las claves del referéndum de autodeterminación del 1 de octubre de 2017 en Catalunya. Anuari del Conflicte Social, 7, 1-32.

López Meri, A. (2016). Twitter-retórica para captar votos en campaña electoral. El caso de las elecciones de Cataluña de 2015. Comunicación y Hombre, 12, 97-118.

Martí, D., \& Cetrà, D. (2016). The 2015 Catalan election: a de facto referendum on independence?. Regional and Federal Studies, 26(1), 107-119. https://doi.org/10.1080/13597566.2016.1145116

Mazzoleni, G., \& Schulz, W. (1999). "Mediatization" of Politics: A Challenge for Democracy?. Political Communication, 16(3), 247-261. https://doi.org/10.1080/105846099198613

Medina, L. (2014). Més partits i més polarització: els canvis en la competència electoral a Catalunya. Barcelona: Institut de Ciències Polítiques i Socials (IPCS).

Meyer, T. M., Haselmayer, M., \& Wagner, M. (2017). Who gets into the papers? Party campaign messages and the media. British Journal of Political Science. https://doi.org/10.1017/S0007123417000400

Muñoz, J., \& Tormos, R. (2015). Economic expectations and support for secession in Catalonia: between causality and rationalization. European Political Science Review, 7(2), 315-341. https://doi.org/10.1017/S1755773914000174

Palau-Sampio, D., Carratalá, A., \& Valera-Ordaz, L. (2017). Mediatización y encuadres de campaña: Análisis comparado de la información de partidos y medios en las elecciones generales de 2015. El profesional de la información, 26(4), 602-610. https://doi.org/10.3145/epi.2017.jul.04

Paniagua Rojano, F. J. (2004). La nueva comunicación electoral en España. Revista latina de comunicación social, 7(58).

Patterson, T. (1993). Out of order: How the decline of the political parties and the growing power of the news media undermine the American way of electing presidents. New York: Alfred Knopf.

Peris Blanes, A., Llorca-Abad, G., Sánchez Castillo, S., \& López-Rico, C. M. (2017). La televisión y la formación de opinión pública: informativos y debates electorales. In $\mathrm{G}$. López-García, G. \& L. Valera-Ordaz, L. (Eds.), Pantallas electorales: el discurso de partidos, medios y ciudadanos en la campaña de 2015 (pp. 101-128). Barcelona: Editorial UOC.

Rodríguez-Virgili, J., López-Escobar, E., \& Tolsá, A. (2012). La percepción pública de los políticos, los partidos y la política, y uso de medios de comunicación. Communication \& Society, 24(2), 7-39.

Sabucedo, J. M., Barreto, I., Seoane, G., Alzate, M., Gómez-Román, C., \& Vilas, X. (2017). Political Protest in Times of Crisis. Construction of New Frames of Diagnosis and Emotional Climate. Frontiers in psychology, 8, 1568. https://dx.doi.org/10.3389\%2Ffpsyg.2017.01568 
Strömbäck, J. (2008). Four Phases of Mediatization: An Analysis of the Mediatization of Politics. The international journal of press/politics, 13(3), 228-246. https://doi.org/10.1177/1940161208319097

Strömbäck, J. (2011). Mediatization of Politics: Towards a Conceptual Framework for Comparative Research. In E. P. Bucy \& R. L. (Eds.), Sourcebook for Political Communication Research: Methods, Measures, and Analytical Techniques (pp. 367382). New York: Routledge.

Tankard, J. (2001). The empirical approach to the study of media framing. In S. Reese, O. Gandy \& A. Grant (Eds.), Framing public life. Perspectives on media and our understanding of the social world (pp. 95-106). New Jersey: Lawrence Erlbaum.

Trimble, L., \& Sampert, S. (2004). Who's in the Game? The Framing of the Canadian Election 2000 by the Globe and Mail and the National Post. Canadian Journal of Political Science/Revue canadienne de science politique, 37(1), 51-71.

Valentino, N., Beckmann, M., \& Buhr, T. (2001). A Spiral of Cynicism for Some: The Contingent Effects of Campaign News Frames on Participation and Confidence in Government. Political Communication, 18(4), 347-367. https://doi.org/10.1080/10584600152647083

Valera-Ordaz, L. (2018). Medios, identidad nacional y exposición selectiva: predictores de preferencias mediáticas de los catalanes. Reis: Revista española de investigaciones sociológicas, 164, 135-154. http://dx.doi.org/10.5477/cis/reis.164.135

Valera-Ordaz, L., Carratalá, A., \& Palau-Sampio, D. (2017). La batalla de los partidos por la definición de la realidad: los marcos partidistas durante las elecciones generales de 2015. In G. López-García, G. \& L. Valera-Ordaz, L. (Eds.), Pantallas electorales: el discurso de partidos, medios y ciudadanos en la campaña de 2015 (pp. 59-78). Barcelona: Editorial UOC.

Vicente-Mariño, M., \& Campos-Domínguez, E. (2017). Encuadres en tiempos de campaña: diferencias y similitudes entre los perfiles de partidos políticos en Facebook y la cobertura periodística en diarios digitales. In A. Larrondo Ureta (Coord.), Claves del periodismo político y la comunicación estratégica en el escenario comunicativo convergente (pp. 278-298). Bilbao: Gureiker. 